Voix et Images

voixetimages

\title{
Second degré du langage
}

\section{André Brochu}

Volume 40, numéro 3 (120), printemps-été 2015

URI : https://id.erudit.org/iderudit/1032640ar

DOI : https://doi.org/10.7202/1032640ar

Aller au sommaire du numéro

Éditeur(s)

Université du Québec à Montréal

\section{ISSN}

0318-9201 (imprimé)

1705-933X (numérique)

Découvrir la revue

Citer ce compte rendu

Brochu, A. (2015). Compte rendu de [Second degré du langage]. Voix et Images, 40(3), 139-145. https://doi.org/10.7202/1032640ar d'utilisation que vous pouvez consulter en ligne.

https://apropos.erudit.org/fr/usagers/politique-dutilisation/ 


\author{
P O É S I E \\ Second degré du langage \\ $+++$
}

ANDRÉ BROCHU

Université de Montréal

L'un de nos poètes les plus doués pour l'invention de problématiques nouvelles, habile pourchasseur de thèmes sensibles comme de résonances théoriques pertinentes, publie un recueil qui a pour sujet la poésie même, abordée dans ses rapports avec le vécu. Le poème est une maison de long séjour, voilà le titre du dernier livre de Normand de Bellefeuille ${ }^{1}$. Chez d'autres écrivains, le résultat d'une telle démarche d'écriture pourrait être abscons ou indigeste, mais ici, malgré les questions (fort nombreuses, ma foi) qu'on peut se poser, il en va autrement, comme le laisse d'ailleurs entrevoir le sous-titre: Catalogue affectueux 1. Normand de Bellefeuille, comme toujours, cherche à conjoindre recherche intelligente et passion du verbe dans une émouvante synthèse qui bouscule et ravive en nous le sens poétique.

Un aspect toutefois propre à étonner le lecteur concerne la double position du poème, à la fois comme sujet de réflexion et comme pratique d'écriture. Le premier suppose le recours à un langage monosémique, celui même de la science (celle des mots) ou de la théorie. Fréquemment, l'auteur fait du poème l'objet d'une pensée, en rapport avec d'autres objets de méditation intellectuelle tels l'image ou le Sens. Ces concepts pourraient être définis ou explorés en bonne et due prose, comme c'est le cas dans quelques essais récents ${ }^{2}$. Ce qui surprend, $c^{\prime}$ est que le poème comme objet de définition et d'exploration occupe la place du poème au sens deuxième, c'està-dire du texte poétique tel qu'il se réalise à travers une forme particulière, qui exige la polysémie plutôt que le langage univoque. Le poète le dit très bien:

Le poème est de l'autre bord

de la langue

l'équivoque même

à la fois

tendre

et endeuillée (27)

1 Normand de Bellefeuille, Le poème est une maison de long séjour. Catalogue affectueux 1, avec des illustrations de Pierre P. Fortin, Montréal, Éditions du Noroît, 2014, 178 p.

2 Notamment celui de Jacques Brault, Dans la nuit du poème, Montréal, Éditions du Noroît, 2011, 52 p. 
L'équivoque, l'union des contraires (tendresse et deuil), fonde la nature même du texte poétique, qui exprime «non pas le sens mais/le véritable chaos [...]/car le poème est un théorème/toujours irrésolu » (47). Le poème véhicule tout le contraire de l'assertion rationnelle.

Or la formulation d'une telle vérité n'a rien de poétique en soi, et c'est ce qui nous frappe le plus souvent à la lecture des cent quarante-huit textes (poèmes?) du recueil. L'auteur consent volontiers à la prose (déguisée), et même à l'humour pur et simple quand il écrit, sans autre explication:

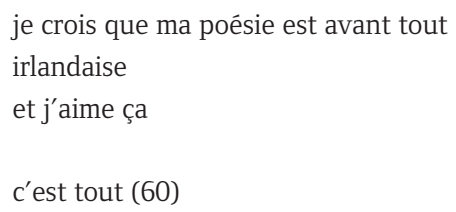

ou encore:

bref

le poème

est mousquetaire (50)

Le ton, souvent déluré ou sans-façon, est à l'occasion celui de la confidence, concernant notamment la mort du père, plusieurs fois évoquée. La confidence s'accompagne parfois d'apostrophes familières lancées au lecteur: « oubliez ça!» (72)

Cela dit, une réflexion complexe se bâtit peu à peu concernant l'essence du poème et, malgré sa dimension nécessairement théorique, elle recourt à l'«équivoque», à la polysémie, dès lors tout à fait de mise. Le poème, par exemple, affiche des rapports privilégiés avec la mélancolie, ce qui fait de lui bien autre chose qu'une machine langagière, et toutes sortes de greffes du vécu contribuent à forger sa riche identité. Langage et existence se fondent en lui:

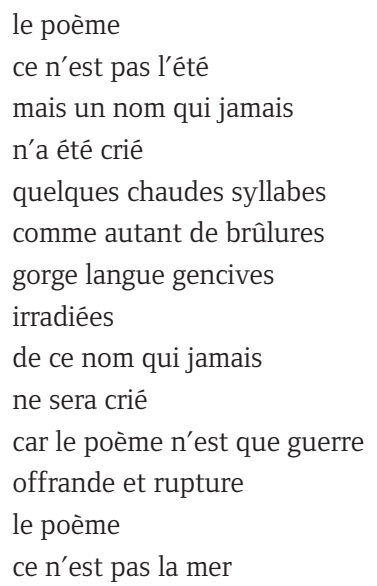


même quand on croit y entendre

la mer (103)

Affaire de "syllabes» et de profération concrète, bref de langage, mais aussi de «guerre/offrande et rupture», donc du vécu le plus intense, le poème est à distance de réalités simples comme l'été ou la mer, incarnations de la beauté du monde, affirme le poète, qui construit ainsi la vérité qui lui est propre.

«Maison de long séjour», dit le titre. On peut s'étonner des choix, tant thématiques que spéculatifs, que nous propose Normand de Bellefeuille, mais son entreprise, si désinvolte puisse-t-elle paraître à l'occasion, provoque en nous un questionnement libérateur.

En même temps que le recueil de Normand de Bellefeuille paraît celui de Pierre Ouellet, aux mêmes éditions ${ }^{3}$. Lui aussi est largement consacré à la poésie. Cependant, De l'air est un livre de prose (parfois nettement poétique, mais pas toujours), qui aborde son sujet en recourant à un style disert et recherché. L'emploi du discours spéculatif étonne moins que les propos en vers du recueil précédent, mais les assertions de Pierre Ouellet ne sont pas pour autant dépourvues d'originalité. Le poème est défini en termes fort imagés, du reste étrangement négatifs:

il est la déposition au creux de la matière la plus vile, la plus basse, la plus vulgaire, celle, rocailleuse, pierreuse, graveleuse, de la langue qu'on parle comme si l'on roulait dans sa bouche un tas de cailloux [...], le dépôt qui fait tache, tartre, limon, sédiment, d'un souffle proprement immatériel. (23)

Très littéraire, tout cela! On remarque que le poème est assimilé à des matières, non à des objets ou à des constructions comme chez De Bellefeuille, qui évoque un «escalier à vis» (57) et une «maison/de long séjour» (85).

Pour Pierre Ouellet, le poème est boue, donc, ou en tout cas complice de la boue, mais il est en même temps et surtout le contraire, il participe de l'air et, par là, de l'«âme perdue de l'ange, du saint, du divin» (27). On retrouve ici l'« équivoque» du poème selon De Bellefeuille, à ceci près que les contraires réunis ont quelque chose de beaucoup plus éloigné et de plus général; ce sont de véritables extrêmes, l'air étant la réalité la plus fondamentale et, sans doute, la plus immatérielle: «Cet air, ce rien» (12). L'extrême positif uni à l'extrême négatif.

Voilà quelques-uns seulement des propos introductifs d'un livre riche en spéculations de toute sorte, qui s'attache non seulement à définir l'indéfinissable poétique, mais aussi à parcourir les domaines où la poésie se réalise, par exemple à célébrer les publications du Noroît (133-135). L'auteur jette aussi un regard rétrospectif sur sa propre production, qui couvre trois genres: poésie, roman et essai (110113), et manifeste un attachement pour celui «qu'on appelle Dieu» et qui répond à

3 Pierre Ouellet, De l'air, avec des illustrations de David Moore, Montréal, Éditions du Noroît, 2014, 186 p. 
«l'improbable nom de poème» (23). Le poème, c'est Dieu, c'est l'air, c'est l'infini bon et mauvais...

La réflexion «poétique» sur la poésie fait appel, chez Ouellet comme chez De Bellefeuille, à des références de haut niveau, mais différentes chez l'un et l'autre. De Bellefeuille cite des auteurs modernes, surtout français: Jacques Derrida (25, 32, 46), e.e. cummings (63), Emmanuel Carrère (74), Christian Bobin (85), Pierre Reverdy (101), Marie-Louise Mallet (111), etc. Ouellet, lui, cite des auteurs de toutes les époques, les littéraires comme les philosophes (Descartes [39], Kojève [44], Heidegger [80]), à commencer par les grands auteurs classiques (Homère [27]) et de nombreuses créations mythologiques ou culturelles (Hadès [17]; Narcisse et Écho [54]; Cythère [65]; Eurydice, Laure, Béatrice [69]). On pourrait en conclure à une inspiration plus métaphysique chez celui-ci, à une sorte de métaphysique naturelle dont l'air est le symbole par excellence; et à un naturalisme métaphysique chez celuilà, ancré dans le quotidien, mais qui s'emploie à transcender l'anecdote de départ.

Une publication ${ }^{4}$ réunit «tous les lauréats et les lauréates du grand prix Québécor du Festival international de la poésie», festival qui a lieu tous les ans à Trois-Rivières depuis 1984. Cet énoncé, tiré du bandeau publicitaire, pourrait laisser entendre que Québécor, le généreux commanditaire, préside depuis le début aux destinées financières de cette noble aventure. En fait, c'est depuis 2004 que l'«empire médiatique» bien connu s'est associé à l'entreprise culturelle trifluvienne.

La formulation du bandeau publicitaire met l'accent sur le côté "gagnant»: cette anthologie est composée uniquement d'extraits des livres qui ont remporté, au Festival de Trois-Rivières, le prix du meilleur recueil de l'année, à l'échelle de tout le Québec. L'enthousiasme est encore plus perceptible dans la préface de Gaston Bellemare, président du Festival: «Ce livre est un livre gagnant issu d'un festival international de poésie gagnant. N'en doutez pas un instant, ce livre saura vous gagner.» (8)

L'anthologie comprend donc des textes tirés de trente recueils, dont quelquesuns du même auteur, sept poètes ayant remporté deux fois le "grand prix Québécor » au fil des ans. Les auteurs, par conséquent, sont au nombre de vingt-trois, et chacun est représenté par trois pages (six pour les «doubles gagnants»). Je dois ici mentionner, par vain souci de transparence, ma présence au nombre des lauréats (en 1995).

Les poètes primés appartiennent tous à la génération des années 1940 et 1950, qui va de Michel Beaulieu (né en 1941) à Serge Patrice Thibodeau (natif de 1959). On n'y trouve aucun représentant des deux grandes générations antérieures, celle des universalistes (Saint-Denys Garneau, Alain Grandbois, Anne Hébert, Rina Lasnier) et celle des fondateurs ou contemporains de l'Hexagone (Gaston Miron, Roland Giguère, Paul-Marie Lapointe, Fernand Ouellette, Jean-Guy Pilon, Gatien Lapointe, Michel van Schendel, Jacques Brault). Les «gagnants», pour bon nombre d'entre eux, ont

4 Gaston Bellemare (dir.), 30 ans de poésie québécoise, Saint-Sauveur-des-Monts, Les Éditions de La Grenouillère, 2014, 136 p. 
été publiés par des maisons d'édition relativement récentes, soit les Écrits des Forges de Trois-Rivières - forcément proches du Festival international de la poésie - et les Éditions du Noroît. Les deux maisons ensemble ont édité plus des deux tiers des livres primés. Le reste se répartit surtout entre l'Hexagone et Les Herbes rouges.

Certes, en raison du hasard des publications et des jurys, et peut-être de certains conflits d'intérêts, tous les auteurs méritants de notre poésie n'ont pas été retenus. Paul Chamberland, France Théorêt, Jean Charlebois, Lucien Francœur, Hélène Dorion et bien d'autres, autant de "gagnants» virtuels qui manquent à l'appel. Mais l'ensemble des trente ouvrages donne une idée juste, encore qu'un peu limitée, de la production poétique de l'époque couverte. Pour chaque poète, trois pages seulement (ou deux fois trois...) présentent l'essentiel. Ces pages sont plus ou moins combles, selon la formule discursive à laquelle le texte a recours. Celles d'un Jean-Paul Daoust, d'un Louis-Philippe Hébert ou d'un Pierre Nepveu sont bien remplies. D'autres, comme les pages de Nicole Brossard ou de Roger Des Roches, offrent plutôt quelques strophes ténues. Bien entendu, la longueur du texte n'a en soi aucune importance, mais quelques mots parfois, si suggestifs soient-ils, ne suffisent pas à rendre justice à toute une œuvre. La concision doit suppléer les silences par la diversité des approches. Bref, le lecteur éprouve un certain sentiment d'inégalité.

L'inégalité apparaît aussi dans le petit nombre de femmes poètes: quatre seulement sur vingt-trois. En plus d'Élise Turcotte, on en compte trois qui sont deux fois primées et qui font entendre des voix importantes: Nicole Brossard, Denise Desautels, Louise Dupré. Cette proportion correspond sensiblement à celle des femmes poètes, pour la même période, dans l'anthologie de Laurent Mailhot et Pierre Nepveu ${ }^{5}$.

Il est impossible de rendre compte totalement d'un tel livre où se succèdent plus de vingt voix différentes. Les sept qui reviennent deux fois sont d'ailleurs bien loin de se répéter. La qualité des vers étant sensiblement la même partout, je parlerai brièvement de quelques textes du début, qui sont le coup d'envoi d'une sélection réalisée à travers une grande diversité de thèmes et de voix.

Le premier poète retenu est Michel Beaulieu, qui meurt l'année même où paraît Kaléidoscope (1985). On trouve, dans les vers cités, un reste de formalisme assez particulier qui privilégie les "graphies sur la page», la «table tout au plus des matières», bref, l'appareil de l'écriture empâté dans une disposition qui «fait allusion aux idées/reçues de l'époque actuelle» (10). La forme se trouve dépassée vers une problématique de l'existence à la fois immédiate et complexe.

Chez Normand de Bellefeuille, qui publie Catégoriques un deux et trois (1986), l'abstraction est première, comme le prouve un tel titre, mais elle se réalise à travers des objets très concrets, souvent d'une trivialité ardente: «Le corps qui meurt a déjà vomi le centre» (15). Tout est intensément matériel, aussi s'agit-il de «désencombrer le monde entier» (16) et d'instaurer, à la place des choses viles, le règne des images, dont le catalogue enjolivera nos «années inutiles» (16).

André Roy (L'accélérateur d'intensité, 1987) introduit un principe spirituel (mais pas nécessairement confessionnel), l'âme, dans son rapport à l'autre: "Mon

5 Laurent Mailhot et Pierre Nepveu, La poésie québécoise. Des origines à nos jours, Montréal, TYPO, coll. «TYPO. Anthologie», 2007, 768 p. 
âme est-elle complète quand je te regarde?» (19) et convoque de grands sujets, comme l'amour, la guerre et la paix, malgré la vision d'un monde semé de «ruines» et du «silence des mouches» (20). Là encore, comme chez les deux écrivains qui le précèdent, l'immatériel vient tempérer la dureté d'une existence livrée au désordre quotidien.

En 1989, une première auteure, Nicole Brossard, signe Installations et $\bar{A}$ tout regard. On peut voir en elle, côté féminin, la figure de proue de sa génération. À la fois féministe et avant-gardiste, elle chante de façon novatrice sa passion, tendre et ardente, pour l'aimée: "viens il n'y a pas de redondance/à ce que poitrine et seins fille ou femme/lèvres et labiales se touchent» (26). On est loin ici de l'abstraction, sinon peut-être d'une abstraction charnelle qui serait l'expression d'un désir transcendant la luxure.

Denise Desautels (Leçons de Venise, 1991), qui a collaboré à de nombreux livres d'artiste, fait volontiers référence aux univers culturels, tout en assumant pleinement le réel. Ce dernier n'est pas de tout repos: «oubliant les faits de vie, l'épuisement, l'abandon, le retrait, l'illusion, la mort [...]/oubliant que j'étais une femme/sur la ligne de feu» (36). La référence à l'art permet de surmonter la vie difficile.

Louise Dupré, dans Noir déjà (1993), fait entendre une voix à la fois plus douce et plus uniformément désespérée: «Toujours la langue/de tant de morts/étonnée/entre les dents/le vide qui s'affaisse/comme un drapé» (43). Le songe mortuaire emplit tout l'espace du vivre, laissant pourtant la chance d'«une extrême raison/ jetée sur le monde/extrême amour» (44).

Il faudrait parler de tous les autres, d'un Claude Beausoleil au lyrisme parfois sombre et parfois chaleureux; de Roger Des Roches et de Jean-Marc Desgent, qui renouvellent l'une par l'autre la syntaxe et l'image; de Pierre Nepveu et de Marcel Labine, qui réinventent le sérieux des choses.

Sans doute aussi doit-on passer vite sur certaines incongruités, comme cet anglicisme probablement voulu: «j'aurai des mains pour l'appui quand tu cantes [penches] de peur» (Pierre Morency [22]; je souligne), ou cette familiarité inhabituelle chez l'auteure: «avec la même main, le même/monde, la même merde/étalée sur la page» (Louise Dupré [114]; je souligne).

Le principal mérite de 30 ans de poésie québécoise n'est sans doute pas de réunir des poésies "gagnantes» - le terme connote plutôt le scintillant monde du sport -, mais de faire entendre les diverses voix d'une génération qui a pris le relais des deux grandes qui l'ont précédée. Sans doute, aucun des poètes récents n'a acquis une célébrité comparable à celle d'un Alain Grandbois ou d'un Gaston Miron. Et aucun ne donne à lire des chefs-d'œuvre comme "La marche à l'amour» (Gaston Miron) ou «La malemer» (Rina Lasnier). Rien là de bien étonnant: on constate le même phénomène ailleurs. En effet, les cent quatre-vingt-sept poètes répertoriés dans le deuxième tome de l'Anthologie de la poésie française du $\mathrm{XX}^{e}$ siècle ${ }^{6}$, qui vont d'André Frénaud à Valère Novarina et qui comptent plusieurs Québécois, semblent inférieurs, à de rares exceptions près, à leurs aînés illustres (Paul Claudel, Paul Valéry,

6 Jean-Baptiste Para (dir.), Anthologie de la poésie française du xx siècle, tome II, préface de Jorge Semprun, Paris, Gallimard, coll. «Poésie», 2000, 684 p. 
Charles Péguy, Guillaume Apollinaire, Paul Éluard, Louis Aragon, Henri Michaux), seuls dignes de nourrir le culte scolaire de la littérature.

Souhaitons toutefois qu'un livre comme 30 ans de poésie québécoise favorise une consécration réelle de notre poésie, malgré l'hiatus des générations. 